Accepted manuscript of paper:

Guttinger, S. (2019). Editing the reactive genome: towards a postgenomic ethics of germline editing. Journal of Applied Philosophy, online first, DOI: 10.1111/japp.12367 Published online: 17 June 2019

\title{
Editing the Reactive Genome: Towards a Postgenomic Ethics of Germline
}

\section{Editing}

Stephan Guttinger*

*Centre for Philosophy of Natural and Social Science, London School of Economics and Political Science, Houghton Street, Lakatos Building, London, WC2A 2AE, UK

E-Mail: $\underline{\text { s.m.guettinger@Ise.ac.uk }}$

\begin{abstract}
The reported birth of genetically modified twins in late 2018 has given new fuel to debates about the ethics of germline genome editing (GGE). There is a broad consensus among stakeholders that clinical uses of GGE should be temporarily banned as the technology is not yet deemed safe for use in humans. However, the idea of a complete ban is dismissed by many based on the expectation that more research will eventually allow scientists to make the technology safe without having to put humans at risk first. In this paper, I will analyse this assumption and argue that it is undermined by recent developments in the postgenomic life sciences. In particular, I will argue that in a postgenomic view of germline editing a complete ban on specific uses of the technology is warranted, because the research needed to assess the safety of these interventions would not be morally defensible.
\end{abstract}




\section{Introduction}

Researchers have been able to modify the genomes of living systems for almost 50 years, but technical limitations meant that precise DNA manipulation could not be achieved in higher eukaryotes such as humans. Only in the last decade (roughly) did targeted genome editing become a widely applicable technology that allows researchers to remove, insert or even re-write specific DNA sequences in almost any organism with great precision. ${ }^{1}$

Scientists usually distinguish between the genetic modification of somatic cells and that of germline cells. Several somatic applications of genome editing technologies are currently being developed ${ }^{2}$ and these interventions are often seen as ethically less challenging a) because of the more local or contained character of the modification and b) because existing ethical and legal frameworks are already in place to deal with such interventions. ${ }^{3}$ Germline genome editing (GGE), in contrast, has been more controversial, mainly because germline cells pass on their genetic material (and hence the modifications they might carry) to future generations. Importantly, as germline modification automatically happens if zygotes are targeted the debate about GGE has primarily focused on the use of genome editing technologies in early-stage human embryos.

GGE has the potential to deliver great benefits but also to create significant harm, both to the targeted individuals as well as to future generations. The risks associated with the use of GGE in humans - combined with reports of first laboratory-based trials in human embryos in $2015^{4}$ - led a range of stakeholders to call for either a complete ban on the use of the technology ${ }^{5}$ or a temporary ban of clinical applications. ${ }^{6}$ Calls for a limited ban of clinical applications have been re-issued and further developed in early 2019 in response to the 
reported birth of genetically modified twins in China. ${ }^{7}$ The Chinese trials, the goal of which was to make the children resistant to HIV infection, were widely condemned by the scientific community, not only because the technology is not deemed safe yet but also because they violated widely accepted ethical and clinical standards. ${ }^{8}$

\subsection{A Prudent Way Forward?}

While at first sight calls for a complete ban seem more radical than calls for a temporary ban, it could be argued that in many ways the latter is the more radical and interesting proposal. Calls for a temporary ban are based on a range of crucial assumptions, in particular a fundamental dichotomy between a research context and a clinical context. The former includes all applications of genome editing in which modified embryos are not implanted, and not allowed to develop beyond 14 days. ${ }^{9}$ The clinical use consists of applications in which modified embryos are implanted and brought to term, thus giving rise to a modified human being that could - potentially at least - further reproduce. Because of the containment (i.e. destruction) of the modified embryos the research use is seen by many as safe. Clinical uses, however, are seen as risk-laden.

A key idea that underlies calls for a temporary ban is that more research, including research uses of GGE, will allow scientists to further develop the technology so that the risks involved with clinical applications could eventually be eliminated or reduced to the point where they become morally acceptable. This research-based approach constitutes what some have called a 'prudent' way forward ${ }^{10}$ as it allows researchers to unleash the benefits of the technology without having to put humans at risk of harm. It is also an exercise in trustbuilding, as researchers want to demonstrate to policy makers and the broader public that 
they are capable of self-regulation and that they can proceed without putting humans and future generations at risk. ${ }^{11}$

But this prudent way forward raises many questions, in particular regarding the role of research and its strict separation from any clinical uses of the technology. Clearly, there are high expectations put on research and the kind of guidance it can deliver on its own. In fact, the whole idea of a temporary and restricted ban of GGE builds on the power of research and its safety. If the risks involved in clinical applications could not be reduced, then there would be no point in calling for a merely temporary ban. And if the research needed to reduce the risks were as risky as the clinical applications themselves then it would again not be possible to make the ban temporary. In fact, in this case the ban would have to be extended to research uses of GGE.

Given the centrality of the concept of a safe and clearly demarcated research context it is important to understand what this context is supposed to entail and deliver. What conditions have to be met to realise a risk-free elimination (or significant reduction) of the risk factors of GGE ${ }^{12}$ And is it indeed possible to have such a safe and hence prudent way forward before any clinical applications of GGE are realised?

Here I want to address these questions by looking at a specific example of a call for a temporary ban of clinical uses. I will first analyse how its advocates portray the benefits of GGE (section 2) and its risks (section 3). In this analysis I will in particular focus on the role assigned to research within the overall pursuit of GGE. I will then argue that many of the conditions that would have to be fulfilled for research to deliver what it is asked to do are 
not attainable because they are built on a picture of the genome that has been undermined by new work in the genomic (or better: postgenomic) sciences (section 4). This, I will argue, undermines the idea that there could be a clear distinction between a safe research context and clinical applications of the technology when dealing with common diseases (section 5). This has important consequences for how we approach GGE and its further development. In a postgenomic world the development of GGE for treating common diseases becomes so fraught with potential risks that its pursuit is not ethically defensible. An exception to this are some cases of rare monogenic diseases and somatic uses of genome editing.

\section{The Idea of a Temporary Ban of GGE}

Calls for a temporary ban of clinical uses of GGE first emerged in 2015 when reports of laboratory-based genome editing experiments in human embryos began to circulate. They were repeated and further specified over the following years in a range of publications, most recently in early 2019 in response to the first clinical uses of the technology in China. ${ }^{13}$

The argument made in these calls is, at first sight at least, relatively simple: GGE should not be used in clinical applications until more research has been done to establish its safety. ${ }^{14}$ This straightforward proposal, however, builds on a range of assumptions, not just about the risks and benefits of the technology but also about the nature of biological systems and the research used to study them. To better understand and assess the proposed temporary bans it is therefore instrumental to first get a better understanding of the different assumptions that underlie them. 
In what follows I will take a closer look at one particular defence of a temporary ban, namely the analysis provided by Gyngell and co-workers in a paper titled 'The Ethics of Germline Gene Editing'. ${ }^{15}$ In line with other positions, Gyngell and colleagues argue against a blanket ban of GGE and advocate a temporary ban on clinical uses of the technology. Their careful argument consists of two parts: first they argue that both the clinical and the research uses of GGE could provide clear benefits for humanity. Second, they claim that these benefits outweigh (or might eventually outweigh) the potential harm caused, as long as more research can establish the safety of the technology when applied in humans. This leads them to conclude that the pursuit of GGE (minimally defined as the research use of GGE and the funding of such research) is not only morally permissible but also morally desirable.

\subsection{The Benefits of Using GGE for Research}

Gyngell et al. highlight several reasons why the research use of GGE is beneficial. One crucial advantage of GGE is that it allows researchers to introduce targeted changes in an embryo's genome. This will allow them to gain deeper insights into the role specific DNA segments and their products (in case open reading frames are targeted) play in early human development. ${ }^{16}$

This will not only provide important benefits for our general understanding of human biology, but it might also help further improve other medical technologies, such as in vitro fertilisation (IVF). As Gyngell et al. highlight, such non-GGE-based clinical applications have become a key driver of the research use of GGE. 
Gyngell and colleagues also highlight that targeted gene editing can be used to create modified human embryonic stem cells that can serve as models for genetic diseases. This allows researchers to work directly with human cells and hence to rely less on the use of (potentially flawed) model organisms. ${ }^{17}$ Modified embryonic stem cells also have the potential to serve as future therapeutic tools for a range of conditions.

These different applications give good reasons for why the research use of GGE should continue, in particular because it poses no or very little risks to humans (as modified embryos can be relatively easily contained). The research use also has an important role to play as an enabler of future clinical applications of GGE. I will return to this point in section 3.1.

\subsection{The Benefits of Using GGE in the Clinic}

When it comes to clinical applications of GGE monogenic diseases (such as cystic fibrosis, Huntington's disease, or haemophilia) are currently seen as the most promising targets of the technology. Scientists already have a relatively good understanding of the genetics of these conditions and the promise is that germline editing will allow researchers to remove or replace the disease-causing DNA sequence in affected embryos thereby making sure that they (and their potential offspring) are free from the disease. Importantly, some of these conditions are difficult or impossible to tackle via somatic gene editing as the latter does not allow researchers to edit the affected sequence in all cells of the organism. This means that germline editing might be the only (or at least the most promising) route to success for a number of these conditions. To date several disease-causing genes have already been targeted by researchers in human embryos using genome editing tools as part of 
(laboratory-based) proof-of-principle studies. An example is a Chinese study that used CRISPR-Cas9 in human embryos to correct the mutation that causes $\beta$-thalassemia. ${ }^{18}$

Some authors have argued that GGE is not needed to deal with monogenic conditions as there are alternative ways for parents to avoid passing on the disease-causing mutation(s) to their offspring. Of particular importance here is the idea that prospective parents can use IVF and preimplantation genetic diagnosis (PGD) to select mutation-free embryos for implantation. ${ }^{19}$ However, it has also been highlighted that this strategy will not work in all cases. If, for instance, both parents are homozygous for a recessive disorder or if one is homozygous for a dominant disorder then genetic selection via IVF/PGD will not be possible. It has also been argued that the IVF/PDG strategy will not always provide doctors with enough viable embryos to select from. ${ }^{20}$ This means that in a few (and arguably rare) circumstances GGE will be the most promising route to success for affected families.

Apart from its use to tackle monogenic diseases, the technology's main and probably broadest appeal arises in the context of 'complex' or 'common' diseases (such as cancer, diabetes or heart disease). Clearly all of these conditions cause significant harm to the people they affect and they also represent crucial challenges for public health systems. The prospect of having a new way of eliminating or at least significantly reducing the risk of these conditions is seen by Gyngell et al. and others as a key reason to support the development and potential use of GGE. 
One aspect that makes it difficult to deal with complex diseases at the genomic level is the fact that more than one stretch of DNA can play a role in their aetiology. To see any positive effects of a genomic intervention it will therefore be necessary to target a range of genomic loci. Dealing with polygenic conditions has so far been beyond the reach of existing approaches (for instance the combination of IVF and PDG or also traditional gene therapy using viral vectors). But, as Gyngell et al. highlight, new gene editing tools now allow researchers to target several genomic loci at once. ${ }^{21}$ This means that, technically at least, targeting complex diseases is becoming a feasible project. ${ }^{22}$

The potential short-term benefits of GGE (the research use and the treatment of monogenic diseases) and the long-term prospects (tackling complex diseases) form the core benefits that pursuing GGE in humans could provide and motivate opposition to a complete ban of the technology. But there are of course also potential costs associated with the pursuit of GGE. One issue that dominates the current debate are safety concerns, in particular the safety of clinical applications of the technology. I will turn to these potential downsides in the next section and highlight why the research use becomes so important in this context.

\section{What is Holding Us Back? Risks Associated with GGE and the Need for More Research}

There are two types of safety issues that come up in the context of GGE: off-target effects and unintended consequences of on-target modifications. The consensus amongst stakeholders and commentators is that these risks have to be eliminated or at least significantly reduced before researchers can move to the clinical context and reap the potential benefits of GGE in humans. ${ }^{23}$ 


\subsection{Off-target Modifications}

A key worry when using genome editing tools in living systems are the so-called off-target effects. By this researchers mean that the intervention could introduce changes in parts of the genome that were not targeted. Such unintended modifications can have devastating effects on the organism as they could, in the worst case, lead to cancer or other severe conditions. Such drastic outcomes might be relatively rare but they are certainly a possibility.

Whilst the last few years have seen vast improvements in the precision of the available editing tools there is still a significant risk that the intervention goes astray and will introduce unintended changes in the DNA. There have been some reports of interventions in human embryos that showed no off-target effects ${ }^{24}$ but these claims have been questioned by other researchers in the field.$^{25}$ Off-target effects are also still an issue in other systems such as Arabidopsis or mice. ${ }^{26}$

Because of the risks that off-target effects pose it is not feasible to pursue a learning-bydoing approach, i.e. to develop clinical applications through cycles of practice and optimisation. This is where the distinction between a research context and clinical applications of genomic interventions becomes central to the debate about GGE. The research context is not only seen as safe but also as a potential enabler of future clinical uses that can help to reduce or eliminate the risks associated with GGE before clinical applications are even attempted. 
This is already being done in the context of off-target modifications. One research avenue that is currently being explored is to find new editing molecules that can perform the same task as existing tools but that can do so with more precision. ${ }^{27}$ Another avenue that is being explored is to optimise the currently used enzymes through a method called 'directed evolution' ${ }^{28}$ This method allows researchers to create and test new variants of an enzyme in vitro, to select the best performer of the batch and to then repeat the whole procedure with this new enzyme using more stringent test conditions. By going through this cycle several times the performance of existing enzymes can be boosted or new functionalities can be introduced over time. This method has already been used to optimise the performance of CRISPR-Cas9, the main tool currently used in genome editing. ${ }^{29}$

Clearly, this path forward relies on more than just the research use of GGE. We have here a general research context that is separated from clinical uses of GGE and that is safe, as no humans are exposed to harm (directed evolution, for instance, is a microbe-based research system). This supports the general idea behind the concept of a temporary ban, i.e. the idea that safe research can be used to eliminate or reduce risk factors that currently make clinical applications not feasible.

It could be objected that this research will not be complete without the use of GGE in human embryos. After all, once new or optimised editing enzymes have been identified they will have to be tested in human embryos to verify their precision in situ. This could mean that the research context is not as safe and unproblematic as the above discussion implies. 
However, the research needed to establish whether off-target modifications have occurred or not does not require that modified embryos are implanted and brought to term. Offtarget effects are created at the point of intervention. This means that modified embryos can be analysed as soon as the editing tools have been deployed (modified cells are usually harvested and analysed $24-48 \mathrm{~h}$ after treatment initiation). There is also no need for longterm follow-up studies as it is the state of the genomic DNA after intervention that is of interest, and not the long-term health of the system as a whole. The point is to find out whether off-target modifications have occurred or not.

This means that research into this safety issue, even if it is conducted in human embryos, can be restricted to a time window of a few hours or days without having to grow the embryo further or even bringing it to term. This reduces the risks this research poses to close to zero (assuming that destructive research on early-stage embryos does not create harm).

At least in the context of off-target effects there is therefore a clear and convincing narrative: through more research scientists will be able to optimise the technology so it becomes less risky. This will shift the cost/benefit analysis in favour of clinical uses as the potential benefits of the applications remain the same whilst the potential costs are reduced. Importantly, this is possible because a clear distinction between a safe research context and clinical applications can be drawn when it comes to dealing with off-target effects.

\subsection{Dealing with Unintended Consequences}


However, off-target effects are only one element of the risk landscape that GGE presents us with. The other key element in terms of safety (ignoring legal and social issues) are unintended consequences of on-target modifications. Introducing changes in the genome, even if the intervention is $100 \%$ precise, can have unintended consequences for the behaviour of the targeted organism. In the extreme case this could lead to the development of disease (e.g. cancer) or negatively affect development and thereby lead to an impairment of the modified person.

The key issue here is a lack of knowledge (rather than a methodological limitation). In most cases, in particular in the context of complex (i.e. polygenic) diseases, researchers simply don't know enough about the genetics of the conditions in order to proceed in a guided and safe manner.

The hope here is, as in the case of off-target effects, that more research will allow researchers to eventually remove or significantly reduce this second roadblock to clinical applications. This path forward again includes more than just the research use of GGE, as it is a broader endeavour that looks at the genetics of disease with a variety of tools, including population genetics, the use of in vitro systems, and animal model organisms.

The two problems this research setting has to solve are a) to tell scientists what genes are involved in a particular condition or could affect the condition so that targets for genome editing can be identified and $b$ ) to provide a better understanding of the biological roles these potential targets play, as they might be involved in more than one process. 
Researchers can only interfere with confidence if they know what a genomic element is doing (and not just that it affects a particular condition).

What they need, then, is a comprehensive map of DNA elements and their functions. The assumption of those calling for a temporary ban on GGE is that the research context (understood more broadly than just genome editing research) can provide such a map without requiring clinical applications of GGE in humans. The prudent way forward is to push ahead with research and to create a map that can guide our genomic interventions before actual modified embryos are brought to term. The question is whether the research needed to create such a map can be as safe and contained as that used to deal with offtarget effects (section 3.1).

\subsection{The Modular Genome}

The idea of the possibility of such a genomic map is partly based on assumptions that have dominated genomics for most of the last five decades. In the traditional picture of the genome it is seen as a material entity (consisting exclusively of DNA) that contains a set of genes. These genes are well-defined entities that can be counted and that have a defined structure and function. Their number and composition are a given that does not depend on the context, which means that genomes can be studied in spatial isolation and independently of developmental time. The map derived from such studies can then be used to guide future research and interventions in the genome.

Researchers of course knew for a long time that the environment of the genome cannot be completely ignored as some genes, for instance, might only be activated under certain 
conditions. But in the old view the environment was not a defining element of what that the genome and its functional elements look like. There was a clear boundary between the genome and what counts as environment.

This picture of the genome and its functioning not only guided large parts of molecular biology in the $20^{\text {th }}$ century but also informed, in part at least, the Human Genome Project (HGP) and its follow-up projects such as ENCODE (The Encyclopedia of DNA Elements). These projects aimed to sequence the human genome (HGP) and to identify and characterise all the genes and other functional elements it contains (ENCODE). The guiding idea was to map the discrete building blocks of the modular genome. ${ }^{30}$

These assumptions also have methodological consequences. With this picture of the genome in hand there is no need to mix clinical applications with the research context. Research into the genome can be done in vitro, in silico, in human cultured cells or in animal model systems. Through this work a map of the genome can be created that can tell researchers what effects specific interventions might have, thereby allowing them to predict which interventions should be safe and which might be potentially dangerous. Human genes and their different effects can be studied without having to bring embryos to term.

However, the neat picture of the genome that underlies such a vision has come under pressure from findings in the (post-)genomic sciences. In the next section, I will have a closer look at these developments. In section 5, I will discuss what this means for the sharp distinction between a research and a clinical context. 


\section{The Reactive Genome}

Research in what are now often called the 'postgenomic' life sciences (roughly defined as the research that followed the completion of the HGP in 2003) ${ }^{31}$ has fundamentally reshaped our understanding of the genome and its functioning. ${ }^{32}$

There are three extensions to/transformations of the old picture of the genome that I want to highlight here. The first transformation relates to the level of nucleotide sequence itself: whereas for a long time the genome was thought of as an assembly of genes (connected by large stretches of non-functional or 'junk' DNA) the HGP and the work done in its wake (in particular the ENCODE project) radically changed this understanding. ${ }^{33}$ As genomicists started to realise that there are fewer genes in a human genome than originally thought they also found that the majority of non-coding sequences have functional significance and could be central to the development and health of the organism. ${ }^{34}$ This means that the number of relevant 'parts' of the genome was radically expanded. Moreover, the ENCODE project has shown that there isn't just 'the' set of functional elements in a genome. Depending on the context, different cells contain different networks of functional elements. ${ }^{35}$ This has led ENCODE researchers to question the idea of 'completeness' when it comes to creating a map of functional elements. ${ }^{36}$

All of these new insights also change our understanding of how DNA manipulation could affect the health and development of an individual: in the old picture interfering with noncoding DNA might not have been considered problematic; it was all about interfering with genes and their immediate regulatory elements. Now such interventions obtain a new 
significance as they target sequences that are not just 'junk' but that could be functional, depending on the context.

The second transformation I want to highlight here included an even more radical extension of the elements that are deemed relevant parts of the genome. This transformation is closely linked to research in epigenetics (a field that pre-dates the HGP but which made significant advances in the post-HGP era). Key to molecular epigenetics is the idea that there can be changes to gene expression (and hence the phenotype of an organism) without changes to the DNA sequence. Crucial factors that become part of the genome's functioning are methyl groups (covalently attached to cytosine nucleotides) and histone proteins and their modifications (for instance acetylation, methylation or sumoylation).

What is important about this second development is that the number of entities that form part of a functional genome was extended beyond mere nucleotides; methyl groups and histones came to be seen as relevant parts of the genomic system. By that the spatial boundary of the genome became extended to such an extent that it has become difficult to define the term. ${ }^{37}$ The notion of "the" genome became much vaguer as a result.

This spatial extension of the genome is also significant because it became part of a third transformation of how scientists picture the genome. Up to the 1990s, the genome was largely thought of as a timeless entity, a stable set of genes that then shape how the organism develops over time. Even though it was known that DNA might accumulate mutations over time and that genomes - thought of as part of lineages - are shaped by 
evolution, at the level of the individual organism the genome was considered to be a welldefined and largely timeless entity. ${ }^{38}$

However, as it became clear that the genome is a multi-component entity that is more than just a set of genes, it also became clear that the structure and functioning of this system is a more complex and dynamic affair. Nowadays the genome is no longer seen as a defined set of genes an individual is born with. It is rather seen as a plastic and developing entity the composition and functioning of which might significantly change over the lifetime of an individual. This also means that the environment now becomes a defining element of what a genome looks like and what it does.

To give an example: depending on the events the body is exposed to the genome might obtain different methylation patterns in an individual, starting with the embryo in the womb (or the culture dish in the case of IVF). These changes will affect how the genome interacts with the rest of the body and how it responds to future inputs. What stretches of the genome are expressed - and what the expressed products look like - is therefore not simply determined by specific genes but by a larger set of entities and processes. As a consequence, the behaviour of the genome (as a dynamic multi-component entity) changes over the lifetime of an individual and depends on its interactions with other processes in the developing body and beyond. ${ }^{39}$ The functioning of the genome, it turns out, is tightly intertwined with its environment (both intra- and extra-cellular) to the point where it becomes difficult to think of the two as clearly separated entities. Nutrition, stress level, genetic dispositions - all of these formerly separate sets of causes become intimately connected. ${ }^{40}$ 
What we are dealing with because of these three transformations is what some authors have called a 'reactive' genome. ${ }^{41}$ This is a dynamic system with vague boundaries, both in a spatial and also a temporal sense.

This also affects how we think about the organism more generally and its interactions with the environment. As Evelyn Fox Keller puts it:

\footnotetext{
"We have long understood that organisms interact with their environments, that interactions between genetics and environment, between biology and culture, are crucial to making us what we are. What research in genomics shows is that, at every level, biology itself is constituted by those interactions - even at the level of genetics". ${ }^{42}$
}

This new understanding of the genome has important methodological consequences for biological research: removing a part of the genome or adding a new sequence is not so much about removing a well-defined module of the system and replacing it with another, as one would manipulate a traditional machine. It is rather like interfering with a complex system of interconnected processes, each depending on the presence and activity of the other. The effects of such interventions can travel far, both in a temporal and a spatial sense. ${ }^{43}$

\section{What Does all of this Mean for the Pursuit of GGE?}


According to defenders of a temporary ban on clinical uses of GGE there is a clear path to ensuring that the benefits of the technology eventually outweigh the risks: by doing more research the key safety issues hampering clinical applications can be removed/reduced, thus making it possible to propose a time-limited rather than a complete ban. A map of functional DNA elements and their physiological importance is central to this plan.

The new picture of the genome that emerges from the postgenomic life sciences creates a problem for the neat research/clinical distinction that underlies this strategy. Because of the plastic and relational nature of the genome, editing the genome at one stage of development can affect later stages of its functioning (and by extension that of the organism). This has fundamental consequences for how research into the safety of such interventions has to be conducted.

To give an example: removing one part of the genome could affect how other parts are methylated. This in turn could have direct consequences for gene expression at later stages in the life of the modified organism. To have such wide-ranging effects the removed part of the genome would not even have to contain coding sequences, i.e. it is not enough to make sure that no essential genes are removed or disrupted by a genome manipulation. The edited part could simply contain a sequence that has regulatory significance for methylation processes at some stage in the life of the modified organism.

Manipulating a particular DNA sequence can affect the future development and health of an organism in new ways as the landscape of stabilised and stabilising processes that make up the biological system could be changed by early-stage genomic interventions. Crucially, 
factors such as nutrition or stress levels, which were traditionally seen as 'outside' or 'nonbiological' factors, suddenly become intimately linked to the genome itself and its modifications. All of these factors have to be taken into account when assessing the possible negative effects of a particular genomic intervention. The reactive genome might show different reaction norms to future events once we change its DNA.

Dealing with a reactive genome therefore asks for a different approach to research: the question of what a particular change in the genome means for its functioning and for the health of the individual can only be addressed by considering a) the whole life cycle of the modified organism and $b$ ) the complex of the individual organism and its environment (if such a distinction is still meaningful). ${ }^{44}$ It is also not enough to just test a modification in specific cell types (liver cells, muscle cells, etc.) as a system-wide analysis of the effects of an intervention is needed (since all cells of the body are modified when using GGE ${ }^{45}$ and since the behaviour of the reactive genome is highly context-dependent). To achieve a reliable estimate of the risks associated with a particular intervention modified human embryos will therefore have to be brought to term eventually and their health monitored to an advanced age. This means that we have to give up the sharp distinction between a safe research context and the clinical use of GGE. It is no longer possible to treat the research context as an unproblematic enabler of the clinical context. In the age of the reactive genome research and clinical applications become fused, including the risks involved.

\subsection{Complex vs. Monogenic Diseases}

As with any absolute statement the above claims have to be qualified. There are certain circumstances in which researchers might be able to uphold a distinction between a safe 
research context and risky clinical applications. I have mentioned one such context above, i.e. the investigation of off-target effects (section 3.1). Another is the case of rare monogenic diseases. What is special about the latter is that the genetics of these conditions are usually relatively simple, as one specific genetic fault is driving the development of the condition, often with no or very little influence from other factors such as lifestyle. This means that these conditions require only single interventions at a specific locus in the genome. In addition, it will often be possible to replace the disease-causing mutation with a known variant that is already present in the healthy population. Human population genetics can therefore give researchers a good insight into the consequences of carrying the replacement sequence.

In the case of complex conditions such as diabetes or heart disease the situation is different. First, the genetics of these conditions is not well understood, meaning that researchers don't have a clear picture of potential targets and the roles they might play in the organism. Second, as complex conditions are polygenic tackling them via genome editing will require a number of edits to see any positive effects of a genomic intervention. Each locus only has a small effect on its own and to see a significant impact on disease risk many loci have to be changed at once.

This not only means that more targets have to be analysed for potential negative sideeffects but also that the genome is rearranged on a larger scale. The number of edits required might range from a dozen to 50 or even hundreds of small and large edits. These edits might not only include single-nucleotide replacements or deletions but also the deletion of larger junks of genomic DNA or the insertion of sequences that are not found in 
the healthy population. Such complex interventions could create cumulative effects that go beyond those of single edits. ${ }^{46}$

Because of the reactive nature of the genome any research into possible side-effects of such complex interventions will require clinical trials in human subjects. Model organisms or in vitro systems using human cells will not be able to give the answers needed as the genomes they carry might display very different reaction norms to the ones in an adult modified organism.

The reactive genome also means that any such study has to be long-term. Researchers need to understand how potential changes affect the reactive genome at any stage of development before they can set free genetically-modified organisms. There is little point in removing a common variant associated, for instance, with the risk of diabetes if the modification turns out to increase the risk of developing early-onset dementia or other serious conditions. This means that modified human embryos not only have to be brought to term but that the modified trial subjects - who cannot consent to being part of such trials - will have to be monitored to an advanced age (without being allowed to further reproduce in order to contain the potentially harmful modifications). Assessing the safety of complex GGE interventions for common diseases will require an ethically highly questionable trial structure. ${ }^{47}$ These trials mean that, in these cases at least, there is no clear line that can be drawn between a safe research context and clinical applications.

\section{Conclusions}


Our scientific picture of the genome is rapidly changing and this also affects how we have to think about dealing with the risks of germline editing. In a postgenomic world establishing the safety of a genomic modification means to study the whole life cycle of the modified organism and its interactions with the environment. This means that the research context will also have to include bringing modified embryos to term, at least in the case of complex interventions. This in turn means that there is no clear boundary anymore between a safe research and a risky clinical context; life, the clinic and research become an interwoven and dense assembly.

As a consequence, the costs associated with developing GGE-based solutions for complex conditions turns out to be too high to justify them. The research context is no longer a safe and innocent enabler of the clinical context, at least not automatically. It might be so in some circumstances, for instance when tackling monogenic diseases or when dealing with the issue of off-target effects. But in the context of common conditions and the complex interventions they require this distinction no longer applies. Once we move towards a postgenomic ethics of genome editing the idea of preventing complex diseases through GGE becomes morally questionable because of the research required to get to that stage.

In a postgenomic world there seems to be little reason to support a merely temporary ban of such clinical applications, as they cannot be obtained without putting humans at significant risk of harm. This suggests a mixed strategy for dealing with GGE in humans: implement 1) a temporary ban for clinical uses of GGE in the context of monogenic diseases and 2) a complete ban for the development of GGE-based treatments of complex diseases. 
This, of course, does not mean that genome editing should not be used to tackle complex conditions. Somatic genome editing remains a powerful weapon and might offer new approaches to dealing with common diseases. Several targeted therapies for cancer are being developed at the moment. ${ }^{48}$ What the above analysis shows is simply that the complex modifications such therapies require should not be permanently written into the human germline.

\section{Acknowledgments:}

I would like to thank John Dupré and the anonymous reviewers of the journal for helpful input on earlier versions of this paper. The initial research leading to this paper has received funding from the European Research Council under the European Union's Seventh Framework Programme (FP7/2007-2013) / ERC grant agreement $n^{\circ} 324186$.

\section{NOTES}

\footnotetext{
${ }^{1}$ Key here was not only the development of CRISPR-Cas9 (Jennifer Doudna \& Emmanuelle Charpentier, 'The new frontier of genome engineering with CRISPR-Cas9', Science 346,6213 (2014): 1258096) but also earlier molecular tools such as zinc-finger nucleases (ZFNs) or 'transcription activator-like effector nucleases' (TALENs) (Thomas Gaj, Charles A. Gersbach \& Carlos F. Barbas, 'ZFN, TALEN, and CRISPR/Cas-based methods for genome engineering', Trends in Biotechnology 31,7 (2013): 397-405).

2 Boris Fehse, \& Ulrike Abramowski-Mock, 'The time is ripe for somatic genome editing: NIH program to strengthen translation', Molecular Therapy 26,3 (2018): 671-674; Natasa Savić \& Gerald Schwank, 'Advances in therapeutic CRISPR/Cas9 genome editing', Translational Research 168 (2016): 15-21.

${ }^{3}$ See, e.g., (Ante S. Lundberg \& Rodger Novak, 'CRISPR-Cas gene editing to cure serious diseases: treat the patient, not the germ line', The American Journal of Bioethics 15,12 (2015): 38-40). However, see (Sarah Polcz \& Anna Lewis, 'CRISPR-Cas9 and the nongermline non-controversy', Journal of Law and the Biosciences 1 (2016): 413-425) for a discussion of specific ethical issues that somatic gene editing brings up.

${ }^{4}$ P. Liang, Y. Xu, X. Zhang, C. Ding, R. Huang, Z. Zhang, J. Lv, X. Xie, Y. Chen, Y. Li, \& Y. Sun, 'CRISPR/Cas9-mediated gene editing in human tripronuclear zygotes', Protein \& cell 6,5 (2015): 363-372.
} 
${ }^{5}$ Edward Lanphier, Fyodor Urnov, Sarah Haecker, Michael Werner \& Joanna Smolenski, 'Don't edit the human germline', Nature 519,7544 (2015): 410-411. See also https://www.nih.gov/about-nih/who-we-are/nih-director/statements/statement-nihfunding-research-using-gene-editing-technologies-human-embryos.

${ }^{6}$ D. Baltimore, P. Berg, M. Botchan, D. Carroll, R.A. Charo, G. Church, J.E. Corn, G.Q. Daley, J.A. Doudna, M. Fenner, \& H.T. Greely, 'A prudent path forward for genomic engineering and germline gene modification', Science 348,6230 (2015): 36-38.

${ }^{7}$ E.S. Lander, F. Baylis, F. Zhang, E. Charpentier, P. Berg, C. Bourgain, B. Friedrich, J. K. Joung, J. Li, D. Liu \& L. Naldini, 'Adopt a moratorium on heritable genome editing', Nature 567, 7747 (2019): 165-168.

8 David Cyranoski \& Heidi Ledford, 'International outcry over genome-edited baby claim', Nature 563,7733 (2018): 607-608; George Q. Daley, Robin Lovell-Badge, \& Julie Steffann, 'After the Storm-A Responsible Path for Genome Editing', New England Journal of Medicine 380,10 (2019): 897-899.

${ }^{9}$ Whether destructive research on human embryos is ethically permissible and desirable is a complex issue that will not be the topic of this paper. Importantly, different countries have different approaches to the issue. In the UK, for instance, destructive research on human embryos can currently be performed to up to 14 days of development (Martin F. Pera, 'Human embryo research and the 14-day rule', Development 144, 11 (2017): 1923-1925). However, there have been calls for the 14-day rule to be relaxed in response to new technological developments (Insoo Hyun, Amy Wilkerson \& Josephine Johnston, 'Embryology policy: Revisit the 14-day rule' Nature 533,7602 (2016):169; Sarah Chan, 'How and Why to Replace the 14-Day Rule', Current stem cell reports 4,3 (2018): 228-34.

${ }^{10}$ Baltimore op. cit.

${ }^{11}$ Sheila Jasanoff, J. Benjamin Hurlbut \& Krishanu Saha, 'CRISPR democracy: Gene editing and the need for inclusive deliberation', Issues in Science and Technology 32,1 (2015): 2532; Daniel Sarewitz, 'CRISPR: Science can't solve it', Nature 522,7557 (2015): 413-414.

${ }^{12}$ Note that there is no consensus amongst experts on what should count as 'significant' in this context.

${ }^{13}$ For an overview of the different positions on GGE that have emerged since 2015 see: K. E. Ormond, D. P. Mortlock, D. T. Scholes, Y. Bombard, L. C. Brody, W. A. Faucett, G. Nanibaa'A, L. Hercher, R. Isasi, A. Middleton \& K. Musunuru, 'Human germline genome editing', The American Journal of Human Genetics 101,2 (2017): 167-176; Christopher Gyngell, Hilary Bowman-Smart \& Julian Savulescu, 'Moral reasons to edit the human genome: picking up from the Nuffield report', Journal of medical ethics (2019): pii: medethics-2018-105084. doi: 10.1136/medethics-2018-105084.

${ }^{14}$ This is a necessary but not sufficient condition. Other conditions that are often mentioned in this context include finding solutions to social and legal issues the technology poses. In this paper I will only focus on the safety issue.

${ }^{15}$ Christopher Gyngell, Thomas Douglas \& Julian Savulescu, 'The ethics of germline gene editing', Journal of Applied Philosophy 34,4 (2017): 498-513.

${ }^{16}$ The power of this approach has been demonstrated by a research team from the Francis Crick Institute in London, which used the gene editing tool CRISPR-Cas9 to delete a particular protein-coding DNA sequence in fertilized human eggs to study the effects this intervention has on early embryonic development (N. M. Fogarty, A. McCarthy, K. E. Snijders, B. E. Powell, N. Kubikova, P. Blakeley, R. Lea, K. Elder, S. E. Wamaitha, D. Kim \& V. 
Maciulyte, 'Genome editing reveals a role for OCT4 in human embryogenesis', Nature 550,7674 (2017): 67-73).

${ }^{17}$ For a discussion of the limitations (and potential) of animal models see Rachel A. Ankeny \& Sabina Leonelli, 'What's so special about model organisms?', Studies in History and Philosophy of Science Part A, 42 (2011): 313-323; Jessica A. Bolker, 'Model organisms: There's more to life than rats and flies', Nature 491 (2012): 31-33. Interestingly, genome editing tools can now be used to further improve existing animal models for disease. For an overview see Lukas E. Dow, 'Modeling disease in vivo with CRISPR/Cas9', Trends in molecular medicine 21,10 (2015): 609-621.

${ }^{18}$ P. Liang, C. Ding, H. Sun, X. Xie, Y. Xu, X. Zhang, Y. Sun, Y. Xiong, W. Ma, Y. Liu \& Y. Wang, 'Correction of $\beta$-thalassemia mutant by base editor in human embryos', Protein \& Cell 8,11 (2017): 1-12.

${ }^{19}$ See, e.g., Eric S. Lander, 'Brave new genome', New England Journal of Medicine 373,1 (2015): 5-8.

${ }^{20}$ Daley op. cit.; Gyngell op. cit.

${ }^{21}$ See, for instance, a study conducted in pigs: L. Yang, M. Güell, D. Niu, H. George, E. Lesha, D. Grishin, J. Aach, E. Shrock, W. Xu, J. Poci \& R. Cortazio, 'Genome-wide inactivation of porcine endogenous retroviruses (PERVs)', Science 350,6264 (2015): 1101-1104.

22 It has to be added here that even with a complete knowledge of the genetics of complex conditions such DNA-based approaches are not guaranteed success. As each of the targeted sequences will only have a small effect on the disease phenotype and as the different modifications might not be simply additive it could well be that even multi-site targeting will not bring about the desired health improvement. On top of that, as Gyngell et al.

themselves point out, there are also environmental factors that influence disease aetiology and which further complicate the picture. See sections 4 and 5 for more on these issues.

${ }^{23}$ Baltimore op. cit.; Lanphier op. cit.; Gyngell op. cit.; Lander 2019 op. cit.

${ }^{24}$ H. Ma, N. Marti-Gutierrez, S. W. Park, J. Wu, Y. Lee, K. Suzuki, A. Koski, D. Ji, T. Hayama, R. Ahmed \& H. Darby, 'Correction of a pathogenic gene mutation in human embryos', Nature 548,7668, (2017): 413-419.

${ }^{25}$ D. Egli, M. Zuccaro, M. Kosicki, G. Church, A. Bradley \& M. Jasin, 'Inter-homologue repair in fertilized human eggs?', Nature 560 (2017): E5-E7.

${ }^{26}$ H. Y. Shin, C. Wang, H. K. Lee, K. H. Yoo, X. Zeng, T. Kuhns, C. M. Yang, T. Mohr, C. Liu \& L. Hennighausen, 'CRISPR/Cas9 targeting events cause complex deletions and insertions at 17 sites in the mouse genome', Nature communications 8 (2017): 15464; Q. Zhang, H. L. Xing, Z. P. Wang, H. Y. Zhang, F. Yang, X. C. Wang \& Q. J. Chen, 'Potential high-frequency off-target mutagenesis induced by CRISPR/Cas9 in Arabidopsis and its prevention', Plant molecular biology 96,4-5, (2018): 445-456.

${ }^{27}$ Mazhar Adli, 'The CRISPR tool kit for genome editing and beyond', Nature communications 9,1, (2018): 1911.

${ }^{28}$ Nicholas J. Turner, 'Directed evolution of enzymes for applied biocatalysis', Trends in biotechnology 21,11 (2003): 474-478.

${ }^{29}$ J. K. Lee, E. Jeong, J. Lee, M. Jung, E. Shin, Y. H. Kim, K. Lee, D. Kim, J. S. Kim \& S. Kim, 'Directed evolution of CRISPR-Cas9 to increase its specificity', Nature communications 9 (2018): 3048.

30 John A. Stamatoyannopoulos, 'What does our genome encode?' Genome research 22,9 (2012): 1602-1611. 
${ }^{31}$ But see (Maurizio Meloni, Political biology: Science and social values in human heredity from eugenics to epigenetics (London: Palgrave Macmillan, 2016)) for a discussion of other meanings of the term 'post-genomic'.

${ }^{32}$ Barry Barnes \& John Dupré, Genomes and what to make of them (Chicago: University of Chicago Press, 2008); Evelyn Fox Keller, 'From gene action to reactive genomes', The Journal of physiology 592,11 (2014): 2423-2429.; Sarah S. Richardson \& Hallam Stevens

(eds.) Postgenomics: Perspectives on biology after the genome. (Duke University Press 2015).

${ }^{33}$ Keller op. cit.; Stephan Guttinger, 'Beyond the genome: the transformative power of functional genomics', (forthcoming).

${ }^{34}$ There was a huge controversy surrounding the ENCODE project and the question of how much of the genome has to be deemed 'functional'. For more on the ENCODE debate see the Supplement in Guttinger, Stephan \& Dupré, John, "Genomics and Postgenomics", The Stanford Encyclopedia of Philosophy (Winter 2016 Edition), Edward N. Zalta (ed.), URL = $<$ https://plato.stanford.edu/archives/win2016/entries/genomics/>.

${ }^{35}$ Nathaniel D. Heintzman, Gary C. Hon, R. David Hawkins, Pouya Kheradpour, Alexander Stark, Lindsey F. Harp, Zhen Ye et al., 'Histone modifications at human enhancers reflect global cell-type-specific gene expression', Nature 459,7243 (2009): 108.

${ }^{36}$ Stamatoyannopoulos op. cit.

37 Guttinger \& Dupré op. cit.; Evelyn Fox Keller, 'Genes, genomes, and genomics', Biological Theory 6,2 (2011): 132-140.

38 Martine Lappé \& Hannah Landecker, 'How the genome got a life span', New Genetics and Society 34,2 (2015): 152-176.

${ }^{39}$ Lappé \& Landecker op. cit.

${ }^{40}$ See Margaret Lock, 'Comprehending the Body in the Era of the Epigenome', Current Anthropology 56,2 (2015): 163-164 and the commentaries on her article for an interesting debate about how epigenetics has undermined the old nature/nurture divide and other boundaries.

${ }^{41}$ Scott Gilbert, 'The reactive genome', in G. B. Muller \& S. A. Newman (eds.) Origination of organismal form: Beyond the gene in developmental and evolutionary biology, MIT Press, (2003), pp.87-101.; Karola Stotz, 'Molecular epigenesis: distributed specificity as a break in the Central Dogma', History and Philosophy of the Life Sciences 28,4 (2006): 527-544.; Keller op. cit.

${ }^{42}$ Keller op. cit., p. 2428.

${ }^{43}$ Becky Mansfield, 'Folded Futurity: Epigenetic Plasticity, Temporality, and New Thresholds of Fetal Life', Science as Culture 26,3 (2017): 355-379.

${ }^{44}$ This change in thinking was not just brought about by epigenetics research but also through work, for instance, in epidemiology where researchers started to study long-term effects of interventions earlier in life. See, for instance, the 'Developmental Origins of Health and Disease' (DOHaD) theory (David J. Barker, 'The origins of the developmental origins theory', Journal of internal medicine 261,5 (2007): 412-417; P. D. Gluckman, M. A. Hanson \& T. Buklijas, 'A conceptual framework for the developmental origins of health and disease', Journal of Developmental Origins of Health and Disease 1, (2010): 6-18).

${ }^{45}$ At least if the problem of so-called 'mosaicism' can be avoided. For more on this phenomenon see, e.g., Daniel Oliver, Yuan Shuiqiao, Hayden McSwiggin \& Wei Yan, 'Pervasive genotypic mosaicism in founder mice derived from genome editing through pronuclear injection', PLoS One 10.6 (2015): e0129457. 
46 Lander op. cit.

${ }^{47}$ It is interesting to note that this need for long-term clinical trials is rarely discussed in the literature on GGE. Most position statements and manifestos simply refer to the need for 'more research', without specifying what exactly this research would have to entail. Two reports that have made this issue explicit are the position statement by the American Society for Gene and Cell Therapy (ASGCT) and the Japan Society of Gene Therapy (JSGT) (Theodore Friedmann, Erica C. Jonlin, Nancy King, Bruce E. Torbett, Nelson A. Wivel, Yasufumi Kaneda \& Michel Sadelain, 'ASGCT and JSGT joint position statement on human genomic editing', Molecular Therapy 23,8 (2015): 1282) and the report by the National Academies of Sciences, Engineering, and Medicine (National Academies of Sciences, Engineering, and Medicine. 2017. Human Genome Editing: Science, Ethics, and Governance. Washington, DC: The National Academies Press. https://doi.org/10.17226/24623). Whilst the former rejects the prospect of clinical trials and supports a 'strong ban' on GGE, the latter leaves the door open for clinical trials under specific conditions.

${ }^{48}$ Chun-Hao Huang, Ko-Chuan Lee, and Jennifer A. Doudna, 'Applications of CRISPR-Cas Enzymes in Cancer Therapeutics and Detection', Trends in cancer 4,7 (2018): 499-512. 